\title{
Panorama das áreas de recepção de resíduos da construção e demolição em Belo Horizonte e municípios limítrofes
}
Overview of the reception areas for construction and demolition waste in Belo Horizonte and bordering municipalities

Panorama de las areas de recepción de desechos de la construcción y demolición en Belo Horizonte y municípios limítrofes

Cristiane F. Pimenta Engenheira Ambiental, Mestranda IFMG cristianepimenta@ambiencia.org

Henrique F. Ribeiro Engenheiro Ambiental, Ambiência Soluções Sustentáveis henrique@ambiencia.org

Samuel O. Pereira Graduando em Arquitetura IFMG Samuel.oliveira@ifmg.edu.br

Neimar F. Duarte Professor IFMG neimar@ifmg.edu.br 


\title{
RESUMO
}

Atualmente, a ineficiência no gerenciamento de resíduos é um dos maiores problemas ambientais enfrentados pela civilização, especialmente no que se refere à falta de locais para destinação dos materiais e às técnicas cada vez mais onerosas para seu tratamento. Neste tema, os Resíduos da Construção e Demolição - RCD se destacam, pois são gerados em grande quantidade e possuem excessivo volume, onerando ainda mais sua destinação. Informações precisas sobre as características e a quantidade de áreas que recebem esse tipo de material ainda são escassas e esses dados são indispensáveis para o planejamento urbano, dos geradores dos resíduos e, ainda, dos investidores do Setor. Visando sanar essa demanda por informações, o presente trabalho pretende apresentar o panorama das áreas receptoras de RCD existentes em Belo Horizonte, municípios limítrofes e Betim, Minas Gerais. $O$ estudo se deu através de informações a respeito das áreas existentes, coletadas nos órgãos ambientais, municipais e estaduais, bem como em empresa atuante no gerenciamento de resíduos de grandes obras, com o intuito de colaborar com o avanço do conhecimento desse grupo de agentes do fluxo dos RCD. Através da pesquisa realizada, foi possível identificar a existência de 32 áreas receptoras de RCD na região de estudo.

PALAVRAS-CHAVE: Resíduos da Construção e Demolição, Áreas Receptoras, Reciclagem, Aterramento, Triagem

\begin{abstract}
Nowadays, the inefficiency in waste management is one of the greatest environmental problems faced by civilization, especially due to the lack of locations for material disposal and increasingly expensive techniques for its treatment. On this theme, Construction and Demolition Waste - CDW stands out because they are generated in great quantity and have excessive volume, making their destination even more expensive. Accurate information on the characteristics and quantity of areas that receive this type of material is still scarce and these data are indispensable for urban planning, by waste generators and even by the Sector investors. Aiming to solve this demand for information, this paper intends to present the panorama of CDW receiving areas in Belo Horizonte, Minas Gerais, bordering municipalities and Betim. The study was carried out through information about the existing areas, collected in the environmental, municipal and state organs, as well as in a company that is active in waste management major works in order to collaborate with the knowledge advance of this CDW flow agents group. Through the survey it was possible to verify It was possible to verify the existence of $32 \mathrm{CDW}$ receiving areas in the region.
\end{abstract}

KEYWORDS: Construction and Demolition Waste, Receiving Areas, Recycling, Grounding, Screening

\section{RESUMEN}

Actualmente, la ineficiencia en la gestión de residuos es uno de los principales problemas ambientales enfrentados por la civilización, especialmente en lo que se refiere a la falta de locales para el destino de los materiales y a las técnicas cada vez más costosas para su tratamiento. En este tema, los Residuos de la Construcción y Demolición - RCD se destacan, pues son generados en grande cantidad y pose en excesivo volumen, haciendo más cara aún su destino. Informaciones precisas sobre las características y la cantidad de áreas que reciben ese tipo de material todavía son escasas y esos datos son indispensables para el planeamiento urbano, de los generadores de los residuos y también de los inversores del Sector. Conel objetivo de sanar esa demanda por informaciones, el presente trabajo intentará presentar el panorama de las áreas receptoras de RCD existentes en Belo Horizonte, Minas Gerais - ciudades limítrofes - y Betim. El estudio se realizó através de informaciones a respecto de las áreas existentes colectadas en los órganos ambientales, municipal y estaduales, así como en empresa actuante en la gestión de desechos de grandes obras con el reto de colaborar con el avance del conocimiento de ese grupo de agentes del flujo de los RCD. Através de la investigación realizada fue posible identificar la existencia de 32 áreas receptoras de RCD en la región.

Palabras Clave: Residuos de la Construcción y Demolición, Áreas Receptoras, Reciclaje, Puesta a tierra, Selección 


\section{INTRODUÇÃO}

A indústria da construção civil possui papel significativo no desenvolvimento econômico e social do Brasil, porém o preço que se paga por isso é alto. Segundo Mália, Brito e Bravo (2011), o peso dessa atividade reflete na esfera ambiental, principalmente devido ao consumo de energia, à extração de matéria prima e ao alto índice de geração de resíduos.

Pinto (1999) estabelece que, a geração per capita dos Resíduos da Construção e Demolição - RCD é entre 0,40 e 0,76 t/(habitante $x$ ano), em alguns municípios brasileiros, o que, de acordo com o Ministério das Cidades e do Meio Ambiente (2006), representa de 50 a 70\% da massa total de resíduos sólidos urbanos.

A grande quantidade dos RCDs gerados torna o seu gerenciamento indispensável, sendo que a ineficiência desse processo é um dos maiores problemas ambientais enfrentados na atualidade, o que é reiterado por Barreira (2005), que define essa problemática como o "Colapso do Lixo", agravado pela falta de locais para destinação dos materiais e pelas técnicas cada vez mais onerosas para seu tratamento.

Castro (2012) afirma que a compreensão das características dos resíduos gerados, associada à combinação das formas apropriadas de disposição final, possibilitam um gerenciamento eficaz, e mostram-se necessárias, diante do fato de existirem diversas formas de disposição com características específicas, como localização e regularização da área, tipo de resíduo recebido, custo de recebimento e tipo de tratamento.

Daher e Fabr (2012) afirmam que, os resíduos provenientes de processos construtivos, reformas ou demolições, quando destinados a locais inapropriados, podem acarretar sérios problemas ambientais. Os geradores, responsáveis pela correta destinação dos resíduos, junto com o poder público, responsável pela destinação dos descartes irregulares, buscam soluções que impeçam a destinação final para locais que não estejam aptos para realizarem suas atividades.

Existem várias dificuldades a respeito do tratamento e destinação final dos $R C D$, sendo que, uma das principais é a carência de informações precisas sobre a quantidade de áreas que recebem esse tipo de material e suas características, dados indispensáveis para o planejamento urbano, dos geradores dos resíduos e, ainda, dos investidores do Setor.

Na Região Metropolitana de Belo Horizonte esse cenário de desinformação não se difere, o que motivou o desenvolvimento do presente trabalho, que pretende apresentar o panorama das áreas receptoras de RCD existentes em Belo Horizonte, municípios limítrofes (Vespasiano, Ribeirão das Neves, Contagem, Ibirité, Brumadinho, Nova Lima, Sabará e Santa Luzia) e Betim que, apesar de não fazer divisa com Belo Horizonte, possui papel importante na região.

Foi possível verificar a existência de 32 áreas receptoras de RCD na região em questão. A maioria desses empreendimentos realiza a atividade de aterramento dos resíduos, sendo que o material que possui mais locais para destinação é o enquadrado na Classe A - Solo e Entulho. Notou-se também que todos os municípios possuem áreas de recepção de RCD e que as regiões da área metropolitana com o maior número de empreendimentos são as Norte, Oeste e Central. 


\section{OBJETIVOS}

O objetivo do presente trabalho é apresentar as áreas receptoras de resíduos da construção e demolição existentes na região limítrofe de Belo Horizonte, bem como suas atividades e localizações, com verificação do tratamento dado aos materiais.

\section{METODOLOGIA}

A escolha dos municípios a serem estudados no presente trabalho foi feita através da verificação da carência de informações a respeito das áreas que recebem RCD em Belo Horizonte. A delimitação dos municípios limítrofes foi necessária, devido à fluidez dos RCD, característica comum em regiões metropolitana onde os resíduos são gerados de forma difusa, permitindo a geração, o transporte, o transbordo e o destino em municípios vizinhos, uma vez que estes se situam muito próximos.

A coleta de dados para o estudo foi realizada da seguinte forma:

- Comunicação, por meio de e-mails, ligações telefônicas e visitas, com as prefeituras dos municípios, através das secretarias que mais se relacionam ao tema, como as Secretarias de Obras e Meio Ambiente.

- As informações repassadas por esses órgãos foram oriundas do conhecimento dos técnicos dos setores contactados, nem sempre sendo formais, de cadastros e registros existentes.

- Comunicação, por meio de e-mails e ligações telefônicas, com a Fundação Estadual de Meio Ambiente - FEAM, órgão responsável pelas licenças ambientais estaduais, através da Gerência de Resíduos Especiais.

- As informações repassadas por esse órgão são referentes aos empreendimentos que deram entrada em processo de regularização ambiental até janeiro de 2016. Como a entrada no processo não garante que os empreendimentos estejam em operação atualmente, foi realizado contato telefônico com os mesmos, para atualização dos dados.

- Verificação das áreas de destinação de RCD gerados em obras de grande porte, cadastradas por empresa especializada em gerenciamento de resíduos (Ambiência Soluções Sustentáveis), no ano de 2016.

Em todas as fontes de dados, foram verificadas as seguintes informações a respeito das áreas receptoras:

- Nome do empreendimento;

- Tipo de atividade exercida;

- Tipo de resíduo recebido;

- Endereço.

Ressalta-se que, inicialmente, a intenção foi a de pesquisar o maior número de informações sobre as áreas, como regularização e dados operacionais. Porém, como se tratam de dados confidenciais, os mesmos não foram disponibilizados pelos empreendimentos.

O contato com a empresa especializada no gerenciamento de resíduos possibilitou, além da verificação do funcionamento das áreas, o entendimento sobre as atividades realizadas, permitindo, assim, uma análise mais precisa da situação desse grupo de agentes do fluxo dos RCD. 
A Resolução CONAMA no 307/2002, e suas alterações, foi sancionada com o intuito de auxiliar nas demandas relacionadas ao gerenciamento dos RCD e estabelece diretrizes, critérios e procedimentos para a gestão dos mesmos, sendo a principal normativa reguladora de todas as etapas do fluxo dos resíduos.

A referida Resolução classifica os possíveis resíduos a serem gerados nas atividades de construção civil, proíbe a disposição em aterros de resíduos sólidos urbanos e em locais não regularizados para este tipo de atividade e estabelece as destinações adequadas para cada classe de resíduos, conforme mostrado no Quadro 1.

Quadro 1- Classificação e destinação dos RCD

\begin{tabular}{|c|l|l|}
\hline Classe & \multicolumn{1}{|c|}{ Descrição } & \multicolumn{1}{|c|}{ Destinação } \\
\hline Classe A & $\begin{array}{l}\text { Resíduos reutilizáveis ou recicláveis como agregados, } \\
\text { inclusive solos provenientes de terraplanagem, } \\
\text { componentes cerâmicos, argamassa e concreto }\end{array}$ & $\begin{array}{l}\text { Deverão ser reutilizados ou reciclados na forma de } \\
\text { agregados, ou encaminhados a áreas de aterro de resíduos } \\
\text { da construção civil, sendo dispostos de modo a permitir a } \\
\text { sua utilização ou reciclagem futura }\end{array}$ \\
\hline Classe B & $\begin{array}{l}\text { Resíduos recicláveis para outras destinações, tais como } \\
\text { plásticos, papel, papelão, metais, vidros, madeiras, } \\
\text { embalagens vazias de tintas imobiliárias e gesso }\end{array}$ & $\begin{array}{l}\text { Deverão ser reutilizados, reciclados ou encaminhados a armazenamento temporário, sendo dispostos de } \\
\text { modo a permitir a sua utilização ou reciclagem futura }\end{array}$ \\
\hline Classe C & $\begin{array}{l}\text { Resíduos para os quais não foram desenvolvidas } \\
\text { tecnologias ou aplicações economicamente viáveis, que } \\
\text { permitam a sua reciclagem ou recuperação }\end{array}$ & $\begin{array}{l}\text { Deverão ser armazenados, transportados e destinados em } \\
\text { conformidade com as normas técnicas específicas }\end{array}$ \\
\hline Classe D & $\begin{array}{l}\text { Resíduos perigosos oriundos do processo de construção } \\
\text { conerão ser armazenados, transportados e destinados em } \\
\text { conformidade com as normas técnicas específicas }\end{array}$ \\
\hline
\end{tabular}

Fonte: Resolução CONAMA no 307/2002 
A Resolução também apresenta algumas definições, conforme mostrado no Quadro 2.

Quadro 2 - Definições relativas aos RCD

\begin{tabular}{|l|l|}
\hline \multicolumn{1}{|c|}{ Discriminação } & \multicolumn{1}{c|}{ Definição } \\
\hline Áreas de destinação de resíduos & São áreas destinadas ao beneficiamento ou à disposição final de resíduos \\
\hline $\begin{array}{l}\text { Aterro de resíduos classe A, de reservação } \\
\text { de material para usos futuros }\end{array}$ & $\begin{array}{l}\text { É a área tecnicamente adequada, onde serão empregadas técnicas de destinação de } \\
\text { resíduos da construção e demolição classe A no solo, visando a reservação de } \\
\text { materiais segregados, de forma a possibilitar seu uso futuro ou futura utilização da } \\
\text { área }\end{array}$ \\
\hline $\begin{array}{l}\text { Área de transbordo e triagem de resíduos } \\
\text { da construção e demolição e de resíduos } \\
\text { volumosos (ATT) }\end{array}$ & $\begin{array}{l}\text { É a área destinada ao recebimento de resíduos da construção e demolição e de } \\
\text { segregados, eventual transformação e posterior remoção para destinação adequada }\end{array}$ \\
\hline Beneficiamento & $\begin{array}{l}\text { É o ato de submeter um resíduo à operações e/ou processos que tenham por } \\
\text { objetivo dotá-los de condições que permitam que sejam utilizados como matéria- } \\
\text { prima ou produto }\end{array}$ \\
\hline Reciclagem & $\begin{array}{l}\text { É o processo de reaproveitamento de um resíduo, após ter sido submetido à } \\
\text { transformação }\end{array}$ \\
\hline
\end{tabular}

Fonte: Resolução CONAMA no 307/2002

Para o presente estudo, foram utilizadas as classificações dos resíduos e das áreas de recepção em concordância com a Resolução apresentada, porém foi verificado que alguns empreendimentos realizam as atividades de triagem/transbordo e aterramento dos resíduos, por isso foi acrescentada a atividade denominada "Aterro Classe A e Área de Transbordo e Triagem - ATT".

Para a organização dos dados coletados, os tipos de atividades exercidas foram classificados da seguinte forma:

- Aterro Classe A;

- Área de Transbordo e Triagem - ATT;

- Aterro Classe A e Área de Transbordo e Triagem - ATT;

- Usina de Beneficiamento.

Os dados obtidos são apresentados no Capítulo 4 - Resultados e Discussão.

A partir dos endereços dos empreendimentos, foi possível a elaboração de um mapa no software ArcGis, para a visualização da distribuição dos mesmos em toda a região.

Salienta-se que, para obtenção das coordenadas para a elaboração do mapa, os endereços dos empreendimentos foram inseridos no Google Maps. Dessa forma, a localização dos mesmos pode apresentar pequena margem de erro, o que não influencia a qualidade do presente trabalho, que tem como intuito analisar a região de forma geral. 


\section{RESULTADOS E DISCUSSÃO}

O Quadro 3, a seguir, apresenta a relação das áreas de recepção de RCD catalogadas na região, através da pesquisa, bem como as atividades realizadas e os tipos de resíduos recebidos.

Quadro 3 - Áreas receptoras de RCD em Belo Horizonte e municípios limítrofes

\begin{tabular}{|c|c|c|c|c|}
\hline Município & Empreendimento & Atividade & Tipo de resíduo & Fonte \\
\hline \multirow{4}{*}{ Belo Horizonte } & Alicerce Empreendimentos Ltda & Aterro CA - ATT & Classe A & Prefeitura \\
\hline & Estação de Reciclagem de Entulho - ERE BR-040 & Usina de beneficiamento & Classe A & Prefeitura \\
\hline & Estação de Reciclagem de Entulho - Pampulha & Usina de beneficiamento & Classe A & Prefeitura \\
\hline & Geo Ambiental Serviços LTDA - ME & Aterro CA & Classe A & Prefeitura \\
\hline \multirow{5}{*}{ Betim } & Alternativa Transportes LTDA & ATT & Classe $\mathrm{A}, \mathrm{B}$ e $\mathrm{C}$ & Prefeitura \\
\hline & AR Locação de Caçambas Ltda & Aterro CA & Classe A & Prefeitura \\
\hline & Gersol Resíduos LTDA-ME & ATT & Classe B & Prefeitura \\
\hline & Prefeitura Municipal de Betim & Usina de beneficiamento & Classe A & Prefeitura \\
\hline & Radial Tratamento de Resíduos Industriais LTDA & Aterro CA & Classe A & Prefeitura \\
\hline Brumadinho & Aterro Sanitário de Brumadinho & Aterro CA - ATT & Classe $\mathrm{A}, \mathrm{B}$ e $\mathrm{C}$ & FEAM \\
\hline \multirow{4}{*}{ Contagem } & Entulhos Ebenezer Itda - ME & ATT & Classe $\mathrm{A}, \mathrm{B}$ e $\mathrm{C}$ & Prefeitura \\
\hline & Gramadus Ltda & ATT & Classe A, B e C & Prefeitura \\
\hline & J \& E Reciclagem & Usina de beneficiamento & Madeira & Prefeitura \\
\hline & Rentall Construção e Empreendimento LTDA & Aterro CA & Classe A & Prefeitura \\
\hline \multirow{2}{*}{ Ibirité } & Reabilitar - Soluções Ambientais LTDA & Aterro CA - ATT & Classe $\mathrm{A}, \mathrm{B}$ e $\mathrm{C}$ & FEAM \\
\hline & Augusto Oliveira Construções e Obras LTDA ME & Aterro CA & Classe A & Prefeitura \\
\hline \multirow{2}{*}{ Nova Lima } & Canadá Caçambas LTDA & ATT & Classe $\mathrm{A}, \mathrm{B}$ e $\mathrm{C}$ & FEAM \\
\hline & Aterro do Mingu & Aterro CA & Classe A & Prefeitura \\
\hline \multirow{2}{*}{ Ribeirão das Neves } & Depósito de Material Excedente & Aterro CA & Classe A & FEAM \\
\hline & Fazenda Córrego Grande/CIF & Aterro CA & Classe A & FEAM \\
\hline Sabará & Empreiteira São Judas Tadeu LTDA & Aterro CA & Classe A & FEAM \\
\hline \multirow{5}{*}{ Santa Luzia } & Associação Educacional Padre Vitor & - & - & FEAM \\
\hline & Czar Serviços Ambientais LTDA & Aterro CA - ATT & Classe A, B e C & FEAM \\
\hline & Empreiteira São Judas Tadeu LTDA & - & - & FEAM \\
\hline & Odeon LTDA & - & - & FEAM \\
\hline & Lafarge Brasil S.A. & Usina de beneficiamento & Gesso & Prefeitura \\
\hline \multirow{6}{*}{ Vespasiano } & Fazenda Cachoeirinha & Aterro CA - ATT & Classe $\mathrm{A}, \mathrm{B}$ e $\mathrm{C}$ & FEAM \\
\hline & Fazenda Maçarico de Cima & Aterro CA - ATT & Classe A, B e C & FEAM \\
\hline & Fazenda Retiro Das Areias & Aterro CA - ATT & Classe A, B e C & FEAM \\
\hline & State Express LTDA/ Transvalente & Aterro CA - ATT & Classe A, B e C & FEAM \\
\hline & DD Transportes e Serviços LTDA & Aterro CA & Classe A & Prefeitura \\
\hline & Lú Terraplanagem TODA - EPP & Aterro CA & Classe A & Prefeitura \\
\hline
\end{tabular}

Como pode ser observado no Quadro 3, foram identificados 32 empreendimentos que recebem RCD na região. Observa-se que o mesmo apresenta os dados disponibilizados pelos órgãos públicos (FEAM e prefeituras). Os dados fornecidos pela empresa especializada em gerenciamento de resíduos foram utilizados para verificação dos empreendimentos que estão em funcionamento.

Não foi possível confirmar a atividade exercida por 3 empreendimentos, mesmo sendo utilizados os dados fornecidos durante a pesquisa e realizadas ligações telefônicas para os próprios empreendimentos. 


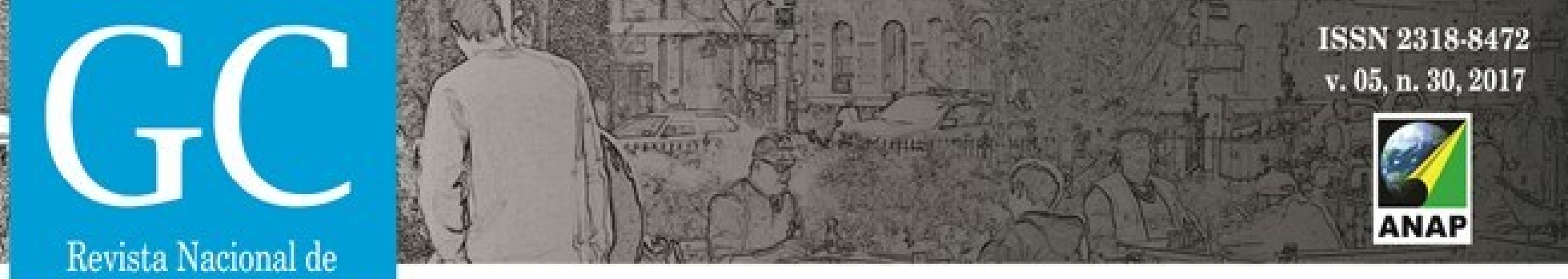

Gerenciamento de Cidades

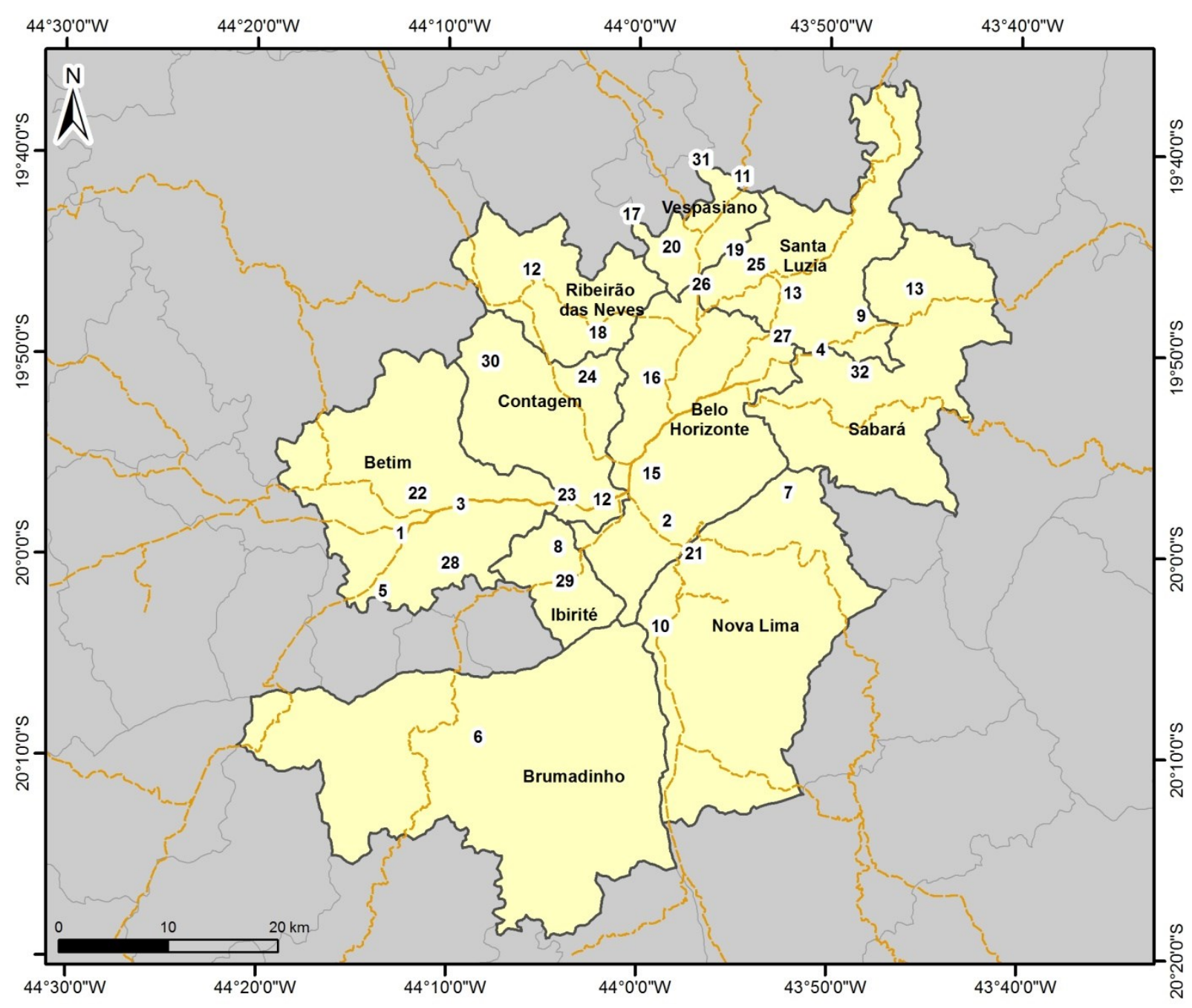

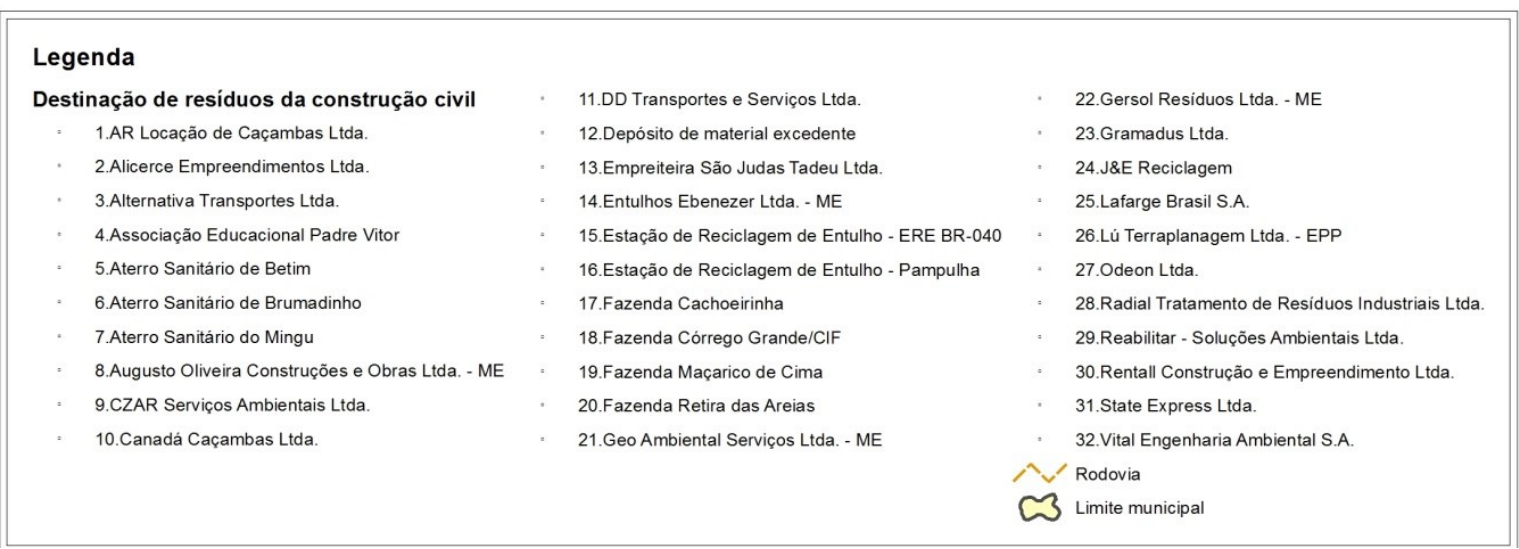

Todos os municípios da região possuem pelo menos 1 área receptora de RCD e os municípios que possuem maior número de empreendimentos são: Vespasiano (6), Betim (5), Santa Luzia (5), Belo Horizonte (4) e Contagem (4). Nota-se também que as regiões Norte, Oeste e Central, abrangidas pelo estudo, são as que possuem maior quantidade de empreendimentos. 


\section{CONSIDERAÇÕES FINAIS}

Através do presente estudo, foram identificadas na região de Belo Horizonte e municípios limítrofes 32 áreas receptoras de resíduos da construção e demolição no ano de 2016. A grande maioria das áreas pesquisadas aterra os resíduos e somente 5 realizam a atividade de beneficiamento.

Salienta-se que, de acordo com o citado no capítulo Metodologia, os dados repassados pelas prefeituras nem sempre foram provenientes de cadastro oficial dos órgãos, sendo, em alguns casos, de conhecimento dos técnicos, o que demonstra uma carência no registro e controle dessas informações. Já em relação aos dados provenientes do Estado, as áreas cadastradas não necessariamente estavam em funcionamento no período analisado e, assim, esse acompanhamento não é realizado pelos órgãos, gerando uma informação imprecisa.

Durante o desenvolvimento do trabalho, houve grande dificuldade para contato com as áreas receptoras de resíduos existentes e também para identificação dos endereços e atividades realizadas, fato que pode dificultar as atividades de planejamento de todos os agentes do fluxo (geradores, transportadores, investidores e poder público).

Diante do exposto, sugere-se que, em futuros trabalhos, além da verificação das áreas existentes no momento da pesquisa, essas sejam visitadas e, dados mais detalhados a respeito de seu funcionamento sejam investigados.

\section{REFERÊNCIAS}

BARREIRA, Luciana Pranzetti. Avaliação das usinas de compostagem do estado de São Paulo em função da qualidade dos compostos e processos de produção, 2005. 204 f. Tese (Doutorado) - Faculdade de Saúde Pública, Universidade de São Paulo, São Paulo, 2005.

BRASIL. Ministério do Meio Ambiente. Conselho Nacional do Meio Ambiente (CONAMA). Resolução no $\mathbf{3 0 7}$, de 05 de julho de 2002. Dispõe sobre a gestão dos resíduos da construção civil e dá outras providências. Brasília, 2002.

BRASIL. Ministério das Cidades; Ministério do Meio Ambiente. Áreas de manejo de resíduos da construção civil e resíduos volumosos. Orientações para o seu licenciamento e aplicação da Resolução CONAMA no 307/2002. 45 f. 2006.

CASTRO, Cristina Xavier. Gestão de resíduos na construção civil, 2012. 54 f. Monografia (Curso de Especialização em Construção Civil) - Escola de Engenharia, Universidade Federal de Minas Gerais, Minas Gerais, 2012.

DAHER, Andrew Motta; FABR, Érika Silva. Gestão de rejeitos de madeira na construção civil: Impactos no Empreendimento Way Pampulha. Pós em revista do Centro Universitário Newton Paiva, Belo Horizonte, Edição 6, p. 151-156, 2012/2.

MÁLIA, Miguel; BRITO, Jorge de; BRAVO, Miguel. Indicadores de resíduos de construção e demolição para construções residenciais novas. Ambiente Construído, Porto Alegre, v. 11, n 3, Júlio/Setembro 2011.

MIRANDA, Leonardo Fagundes; ANGULO, Sérgio Cirelli; CARELI, Élcio Duduchi. A reciclagem de resíduos da construção e demolição no Brasil: 1986 - 2008. Ambiente Construído, Porto Alegre, v. 9, n 1, Janeiro/Março 2009.

PIMENTA, Cristiane Ferreira et al. Quantificação e composição dos resíduos da construção civil em edificações de grande porte. Ares Ambiente \& Resíduos, São Paulo, v. 1, n. 7, p.52-60, 30 nov. 2016. Trimestral.

PINTO, Tarcísio de Paula. Metodologia para a gestão diferenciada de resíduos sólidos da construção urbana. São Paulo, 1999.189 f. Tese (Doutorado em Engenharia) - Escola Politécnica, Universidade de São Paulo, São Paulo, 1999. 
